\title{
Using Infoveillance to Identify Community Concerns/Literacy, Reduce Risk, and Improve Response in Pollution and Health Emergencies
}

\author{
Andrea L. Crampton ${ }^{1, *}$, Nargiza Pearce $^{1}$, and Angela T. Ragusa ${ }^{1}$ \\ ${ }^{1}$ Institute for Land, Water, \& Society, Charles Sturt University, Albury, Australia
}

\begin{abstract}
Contextualised in public health and environmental literacy frameworks, this interdisciplinary research applies the epidemiological concept 'infoveillance' to show how major pollution events can be mitigated by better use of information communication technologies (ICTs). Findings from statistical analysis of Google Trends ${ }^{\mathrm{TM}}$ data during a major Australian air pollution event (2019-2020 Summer bushfires) are presented to compare community environmental and health concerns, as manifested by Google searches, in three affected states. Internet searches related to air purifiers, air quality, and P2 face masks increased as the measured air quality decreased. The commonality of internet search activity found across states, in response to pollution emergencies, presents a valuable, yet underutilised, information source for disaster management and response by health authorities and businesses. Significant correlations between the public's keyword searches and pollution risk suggest Google Trends ${ }^{\mathrm{TM}}$ are an underutilised ICT for identifying public health literacy, needs, and emergency response. The 2019-2020 bushfire infoveillance analysis suggests earlier intervention/awareness of predictable community response to risk-management may mitigate insufficient supply of personal protective equipment (i.e., air purifiers) and alert authorities of illiteracies requiring immediate, urgent ramification to reduce public health risk and improve emergency response, including for vulnerable populations.
\end{abstract}

\section{Introduction}

Air pollution is a growing concern globally [1]. While air pollution derives from multiple and varied sources, 'bushfires', alternatively termed 'wildfires', pose a sizable risk to human, environmental, and economic protection. Information communication technologies (ICT), however, pose new outlets to conceptualise and research how time-sensitive information is delivered, used, and actioned, specifically related to emergency events. This paper presents findings from a study employing 'infoveillance' [2], the use of internet-based information often for epidemiological assessment, to illustrate how and why a collaborative approach between government and public risk knowledge may better mediate emergency action responses to events. Specifically, Australian government air quality data is analysed alongside the public's expression of science/health literacy and risk-management response by information-searching in a global social environment, namely the 'internet'

Air quality monitoring and reporting is deficient in the developed world, including Australia and America [3, 4]. Arguably, less is known, or available, about air pollution to residents in the developing world because of the perception that the State ensures provision of 'clean air' through adequate measurement and legislation, such as the National Clean Air Agreement [5] . Limited public science literacy, coupled with perceptions that the State will suitably protect citizens in emergency events can, and does, leave society 'at risk' when disaster strikes. With internet 'googling' a common contemporary response, large improvements in public literacy and response can be gained from better health authority ICT management and analysis.

Often, it is not until major pollution/health emergency events arise that public (il)literacy becomes evident, and, moreover, becomes necessary to manage or mitigate by prompting, and sometimes enforcing, public health actions. Although this is visible in state governments' response in Australia to enforcing COVID-19 public health-mitigating actions [6], for the Summer 2019/2020 Bushfire Event [henceforth, 'the event'], identifying when and if residents' exposure to pollutants required a personal mitigating action for safety reasons, individual pollution exposure minimisation actions have received little research investigation or government concern [7], despite the life-threatening risk smoke inhalation poses even to those geographically distant from active bushfires.

Worldwide, health authorities' ability to inform and assist the public with the management of unexpected air pollution exposure is lacking [8]. In the absence of such systems, scope exists to utilise ICT generally, and, specifically, the emerging practices of infoveillance, to

\footnotetext{
* Corresponding author: acrampton@csu.edu.au
} 
monitor a population's response to a risk-posing situation. Typically, infoveillance is disease-related, however, in this research, its application is examined for a pollution event.

During the summer of 2019/2020, Australia experienced the worst bushfire season in modern memory. The fires burnt more than 10 million hectares of land [9] and smoke blanketed three states, with the country's capital experiencing levels of harmful particulate pollution. On at least two occasions, the capital experienced some of the highest levels of air pollution in the world, higher than more notorious regions such as Delhi and Beijing [10].The prolonged high levels of particulate pollution from bushfire smoke contributed to the deaths of 417 people and the hospitalisation of more than 4000 [11]. While bushfires and the purposeful burning of land designed to reduce bushfire intensity are a norm in Australia, they do not normally cause such sustained levels of excessive particulate pollution across large geographical areas. As the event unfolded, it became apparent both the general population and health authorities were underprepared to manage the physical and emotional impacts of this sustained pollution event.

While sensitive populations may have some awareness of air pollution risks and measures to reduce personal exposure, the majority of the population was, arguably, unaware of the risks posed by particulate matter pollution, how to interpret air quality information to gauge personal risk, and/or ways to reduce personal pollution exposure. For many, their science/health information deficit led them to the internet and, most likely, to Google [12]. As a common port of call for public health information [13-15], Google also has the capacity to serve as a rich source of information for those that serve the community. Using Google Trends ${ }^{\mathrm{TM}}$, one of the most popular tools for identifying relative internet search volumes (RSV) [16], it is possible to see changes in community search patterns during the course of an event and thus note potential issues of common concern, including confusion.

The potential of Google Trends ${ }^{\mathrm{TM}}$ data to better equip public health officials has been demonstrated for influenza outbreaks in America [17], COVID-19 [16], and Dengue fever in Indonesia [18], where traditional surveillance data has a 1-2 week lag, but internet trend data indicating public searching of symptoms and other related concerns that can be reviewed daily. In this instance, internet trend data can help authorities improve resourcing to areas where area-specific health data is unavailable to improve officials' ability to respond more accurately to demographic and agent-specific surveillance data. Google Trends ${ }^{\mathrm{TM}}$ data also has shown promise for enhancing communication about new cancer screening tools by identifying changes in public internet searches after the release of initial information [15]. ICT, as an information tool, also holds potential to inform Australian authorities of public concerns about pollution, as Google Trends ${ }^{\mathrm{TM}}$ data already has been used by tourism bodies in China to assist with marketing tourism opportunities in regions of potential concern to travellers [19].

If infoveillance is implemented at the beginning of a major pollution event, results also could be informative to authorities and industries seeking to identify what resources may need to be prioritised as the event progresses. In this analysis, the resource focus is two consumer goods that demonstrate public interest in translating their air quality knowledge and perception of personal risk into health-mitigating actions, such as further investigating or purchasing recommended consumer goods (i.e., air purifiers or P2 masks). This ICT-mediated activity could be viewed as an increase in the level of individual health literacy from functional to interactive as articulated by Nutbeam [20]. Although assessing individual shifts from functional health literacy (risk-awareness) to interactive health literacy (risk-action) lies beyond the scope of the present study, heightened consideration of Google Trends ${ }^{\mathrm{TM}}$ data may offer a more informed opportunity to modify and adjust communication actions demonstrated effective for raising health literacy [21]. Methodologically, primary data sourcing methods such as surveys and focus groups typically used to obtain/assess public knowledge or opinion are unlikely to be possible during emergency events. Hence, infoveillance generally, and Google Trends ${ }^{\mathrm{TM}}$ data specifically, offers a timely and efficient mechanism in crises events for officials and industries to better anticipate, and possibly attenuate, public panic behaviour, such as panic consumer purchases resulting in shortages of essential products/services that, in part, may reflect decreased science/health literacy than 'real' action needs.

\section{Methods}

Secondary data analysis was employed to answer three research questions:

1.Did hazardous smoke events in three Australian states (New South Wales (NSW), Victoria (VIC), and the Australian Capital Territory (ACT)) during the November, 2019-February, 2020 'Summer Bushfire Event' precipitate changes in residents' internet searches for the terms 'air quality', 'air purifier', or P2 'masks'?

2. Did residents' location affect their Google searches?

3. Is there a relationship between measured/personally experienced hazardous air quality and residents' internet search activity for these terms?

Collectively, these questions contribute to the broader research aim: to identify if conducting infoveillance analysis of Google Trends ${ }^{\mathrm{TM}}$ data may alert public health authorities, and inform emergency response activities, of key science illiteracy areas and mitigation actions residents explored for an event (i.e., air pollution). To achieve this broader research aim, the research design investigated residents' internet searching for terms provided in government health advice. Three hypotheses were created and tested:

i. Google Trends ${ }^{\mathrm{TM}}$ data will show increased search activity for emergency event-related terms when the event reaches closer physical proximity to the public due to (real or perceived) heightened risk.

ii. Government public health terminology recommendations will manifest in increased Google searches by residents. 
iii. As 'new' locations experience 'the event', similar Google searching behaviour will be revealed by Google Trends ${ }^{\mathrm{TM}}$ data if wider health/science illiteracy remains and/or public needs are similar.

If these hypotheses are confirmed, then infoveillance analysis may be possible to better predict public science/health information and action-mitigating 'needs' before an emergency (i.e., bushfire, pandemic, etc.) reaches new locations.

Three Google search terms were chosen for this investigation because of their alignment with the Australian Government's general advice for decreasing residents' hazardous smoke exposure during a pollution event. The first search term was 'air quality' due to Government advice that residents check official air quality measures to learn about 'who' should stay indoors [22-25]. Second, 'P2 masks' was chosen because of official advice that wearing a $\mathrm{P} 2$ mask reduces hazardous smoke exposure [22, 23, 25]. Selection of the third search term, 'air purifier', reflected Government advice, particularly for remote/rural residents, that purchasing air purifiers for personal use may improve indoor air quality where residents lacked access to public spaces with commercial air conditioning [22, 24-25]. Additionally, a control search term, 'gloves', was included to determine if search patterns manifested by Google Trends ${ }^{\mathrm{TM}}$ for the tested search terms were unique, rather than reflecting a broader set of trends around personal protection actions unrelated to air quality. The inclusion of a control term mirrors methods others have used to identify trends relevant to a specific event, verses trends produced for other reasons [17].

The Google Trends ${ }^{\mathrm{TM}}$ data timeline was determined first by reviewing externally validated air quality data relative to small particulate matter (PM2.5) reported publicly on the www.apicn.org website for the central business districts (CBD) in the three research sites. PM2.5 poses a significant health hazard and is a core constituent of smoke measured by state environmental agencies as part of the Air Quality Index (AQI). AQI PM2.5 levels between 100-150 are considered unhealthy for sensitive groups and $>150$ are unhealthy for everyone.

Data collection entailed two steps. First, AQI data for VIC was drawn from the monitoring station for Melbourne CBD. For NSW, the Point Cook reader (closest to CBD) was sampled and for ACT, the Canberra Civic readings were used. The sampling timeframe (1 November 2019 - 25 January 2020) was chosen using two selection criteria: a. the sampling timeframe captured when the AQI for PM2.5, according to the 2016 American Environmental Protection Agency's standard, first exceeded 50, a level considered 'moderate', but posing a health risk to sensitive groups, on two consecutive days, across each research location; b. the timeframe excluded the onset of the COVID-19 pandemic in Australia. This second criteria served as an additional external validity measure by ensuring the behaviour manifested, public Google searching for P2 masks, existed in relation to the research focus (the Summer 2019/2020 Australian bushfire event) and not in response to the emerging COVID-19 pandemic, despite high PM2.5 pollution levels persisting beyond the data collection period terminating in mid-January, 2020. As a control for AQI data, and to permit seasonal comparison with the 2019/2020 bushfire event, AQI summer measurements also were collected for each location for the same dates in 2018-2019. Second, the 'relative search volume' (RSV) from Google Trends ${ }^{\mathrm{TM}}$ data was extracted for each search term, by research location and summer timeline, into an Excel spreadsheet.

Data analysis commenced by comparing the Google search data with the air quality data for each location and timeframe to test each hypothesis. Spearman Correlations were run, using the program $\mathrm{R}$ commander (version 2.53 ), at $95 \%$ confidence intervals (CI). These methods align with Husnayain, Fuad, and Lazuardi's (2019) comparison of dengue cases and dengue-related Google searches, Effenberg et al.'s (2020) research of COVID-19 cases and Google searches, and Schootman et al.'s (2014) exploration of the relationship between Google searches for cancer screening and actual screening rates. Further, as Mavragani et al.'s (2018) review of Google Trends ${ }^{\mathrm{TM}}$ use in public health notes, correlation analysis is both a prevalent and suitable method for such secondary data analysis investigations.

\section{Results}

NSW was the first state to experience prolonged smoke exposure during the event, followed by VIC, and, lastly, the ACT.

Fig. 1 shows AQI data for the control and event periods to document, and externally validate, the event's severity in the three research locations. In NSW, PM2.5 first exceeded an AQI of 50 for 2 consecutive days on 13 November 2019, with a reading of 113.5. Over the next 2.5 months, there were only 15 days where NSW's AQI level for PM2.5 was below 51 and 18 days where levels exceeded 100. VIC experienced the least days of elevated PM2.5. For 8 days commencing 21 November 2019, VIC recorded its first set of two days with an AQI of PM2.5 above 50. VIC had fewer days of prolonged hazardous smoke than ACT, with 17 days experiencing PM2.5 levels above 50 during the 2 months that followed the first event. Four of these days had a consecutive period where the PM2.5 AQI exceeded 100, including one day with a peak of 272 . The ACT experienced the longest period with hazardous air quality. ACT experienced its first two consecutive days where PM2.5 exceeded 50 on the $9^{\text {th }}$ and $10^{\text {th }}$ of December 2019, with AQI values of 149 and 173 respectively.

This was the beginning of an eight-day period where AQI PM2.5 readings exceeded 90. Between 9 December 2019 and 6 February 2020, ACT residents experienced a prolonged exposure to hazardous air quality, with only 6 days where PM2.5 levels dropped below 50 and 6 days where it exceeded 200. Further, this pollution event resulted in several days of the ACT recording the highest air pollution levels in the world [10] at 549 on 2 January 2019 and 714 on 6 January 2020. 


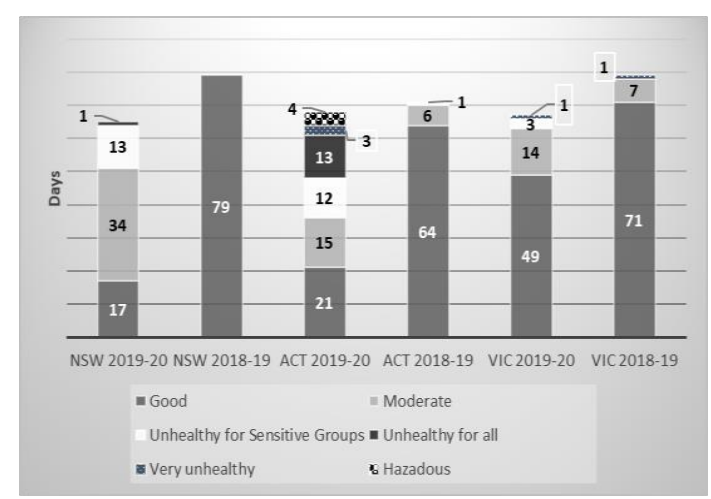

Fig. 1. Control/event comparison of PM2.5 by state.

Google Trends ${ }^{\mathrm{TM}}$ data for the three states (Fig. 2-4) support Hypotheses 1-3, with some geographical variation. Generally, as the event increased, demonstrated by the AQI rise in PM2.5, residents in the corresponding areas started internet searching for event-related terms, evidencing support for Hypothesis 1. Specifically, searches for 'air quality' and mitigating actions aligned with Government advice, utilisation of 'P2 masks' and 'air purifiers'. This finding lends support to accept Hypothesis 2. An anomaly, however, becomes visible when these states are compared. In NSW, increased PM2.5 initially did not yield high RSV for any eventrelated term. RSV alignment between poor AQI and increased internet searching occurred mid-December 2019 for the three search terms investigated (Fig. 2). In contrast, VIC and ACT Google Trend data showed closer alignment between event exposure and increased eventrelated RSV (Fig. 3-4).

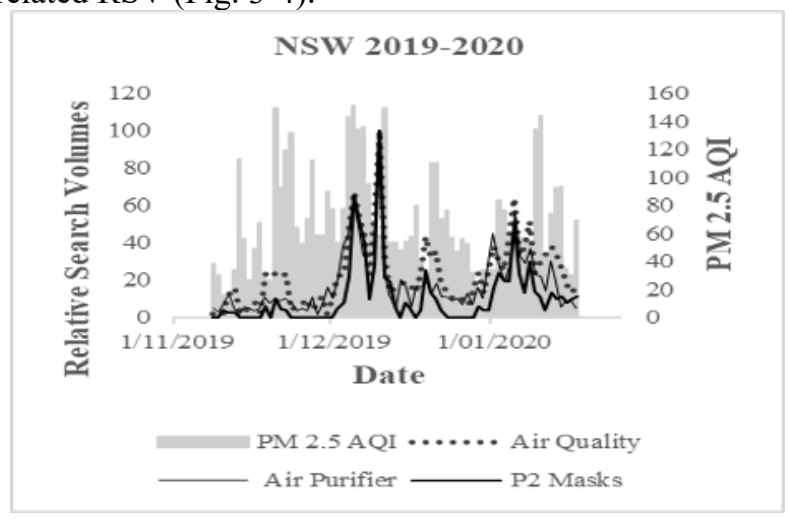

Fig. 2. NSW Comparison PM2.5/ Google Trends ${ }^{\mathrm{TM}}$ RSV.

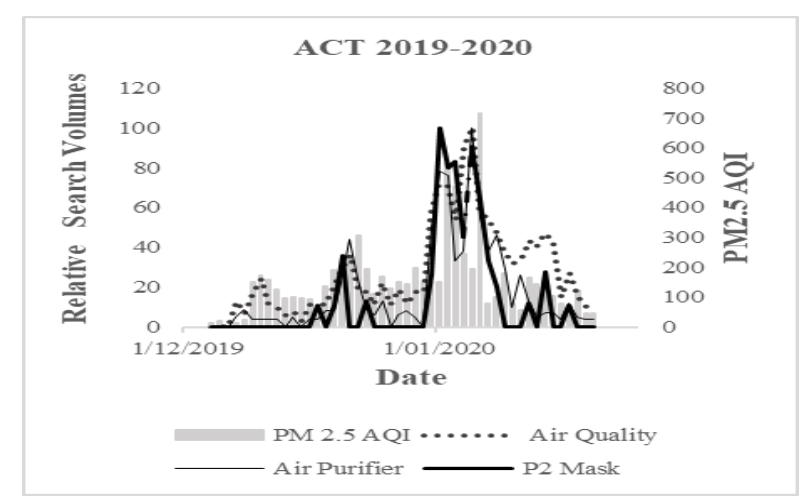

Fig. 3. ACT Comparison PM2.5/ Google Trends ${ }^{\mathrm{TM}}$ RSV.
In VIC, air purifier searches were slightly elevated before the event, yet remained at a baseline level for that term by not exceeding an RSV of 15 until several days of exposure to PM2.5 above 50 (Fig. 4). Consistent with NSW and ACT's increased action-mitigation term searching, VIC's P2 mask and air purifier searching aligned with heightened PM2.5, albeit occurred almost simultaneously in December 2019 after multiple days of prolonged PM2.5 exposure above 50. This finding lends support to accept Hypothesis 3.

Increased ( $>15 \mathrm{RSV})$ searches for P2 masks and air purifiers commenced several days after searches for air quality and prolonged exposure to increased PM2.5 in NSW and VIC (Fig. 2-3). In contrast, VIC residents commenced internet searching for air purifiers prior to searching for masks and air quality, as well as prior to physically experiencing the event (Fig. 4).

RSV of the two action-mitigating terms, P2 masks and air purifies, by location and date further lends support for Hypothesis 3, suggesting infoveillance may serve a useful predictive capacity for identifying public health literacy and goods/action needs in locations about to experience the same, or similar, event. Fig. 5-6 illustrate search trends for air purifiers and masks in each state not only generally followed periods of increased PM2.5, they also exhibited similar science literacy/action needs across locations. If infoveillance was conducted when the first state (NSW) experienced the event (early December 2019), then officials would have been able to anticipate future locations' science literacy/action needs for air purifiers and P2 masks (i.e., ACT and VIC midDecember 2019 - January 2020) and additional NSW needs in the 'second' peak event (January 2020).

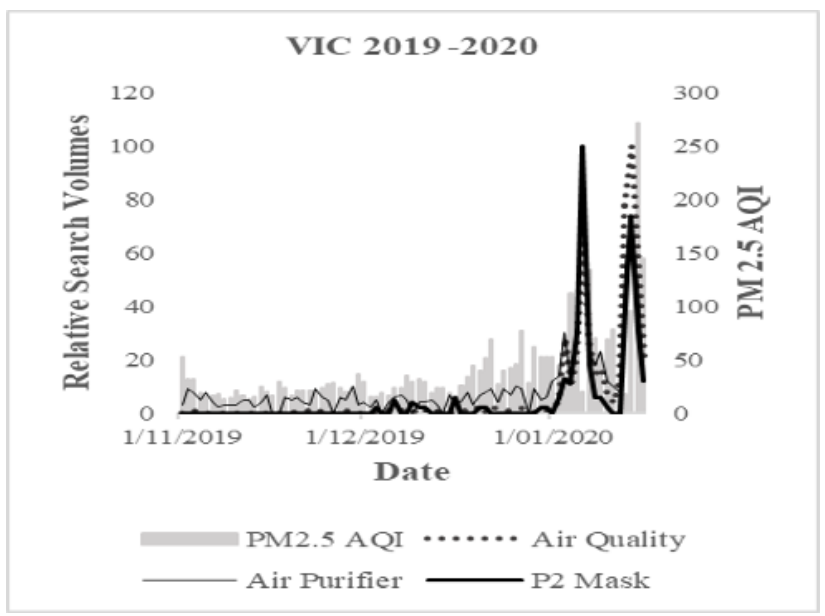

Fig. 4. VIC Comparison PM2.5/ Google Trends ${ }^{\mathrm{TM}}$ RSV. 


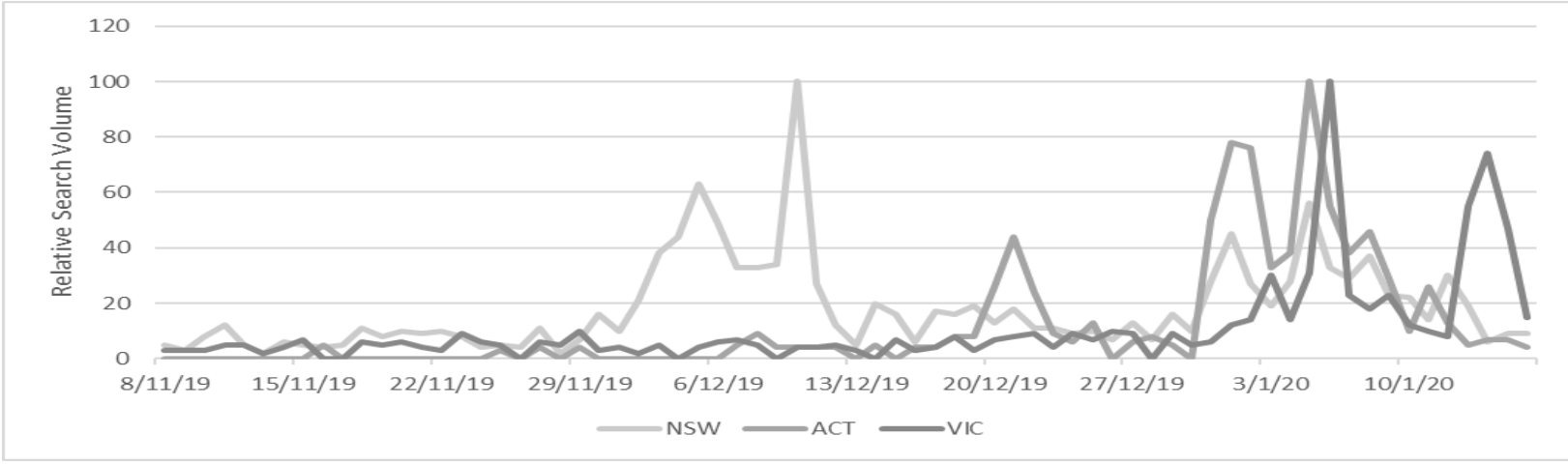

Fig. 5. Air Purifier Google Trends ${ }^{\mathrm{TM}}$ RSV by state and event timeline.

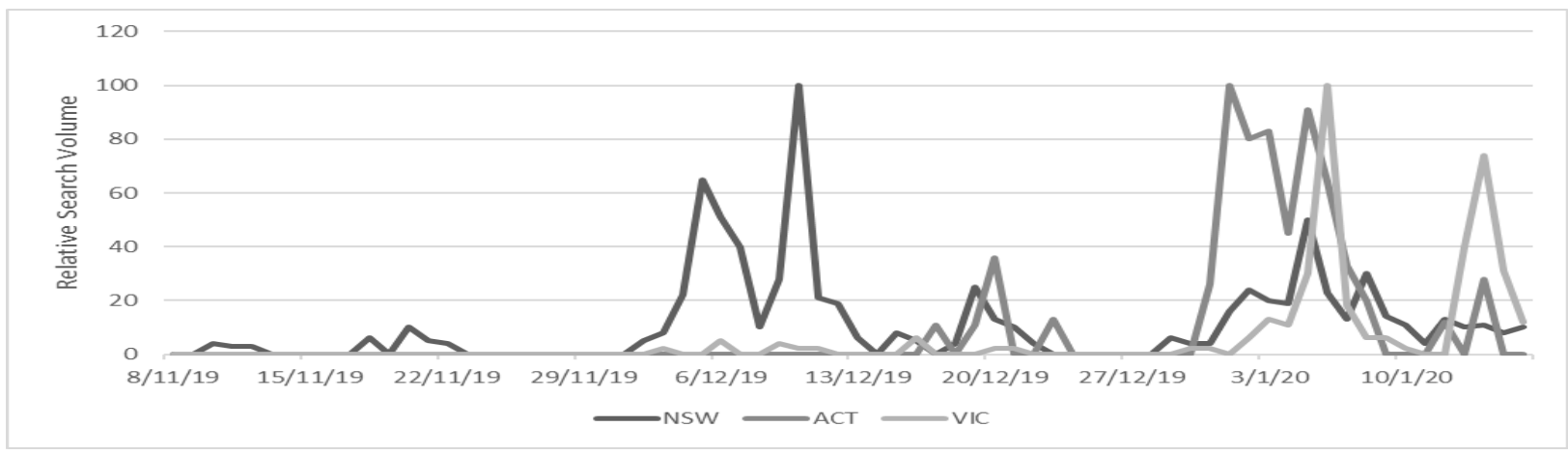

Fig. 6. P2 Mask Google Trends ${ }^{\mathrm{TM}}$ RSV by state and event timeline.

In contrast, the control term 'gloves' failed to display trends with the event (Fig. 7) or achieve statistical significance (Table 1).

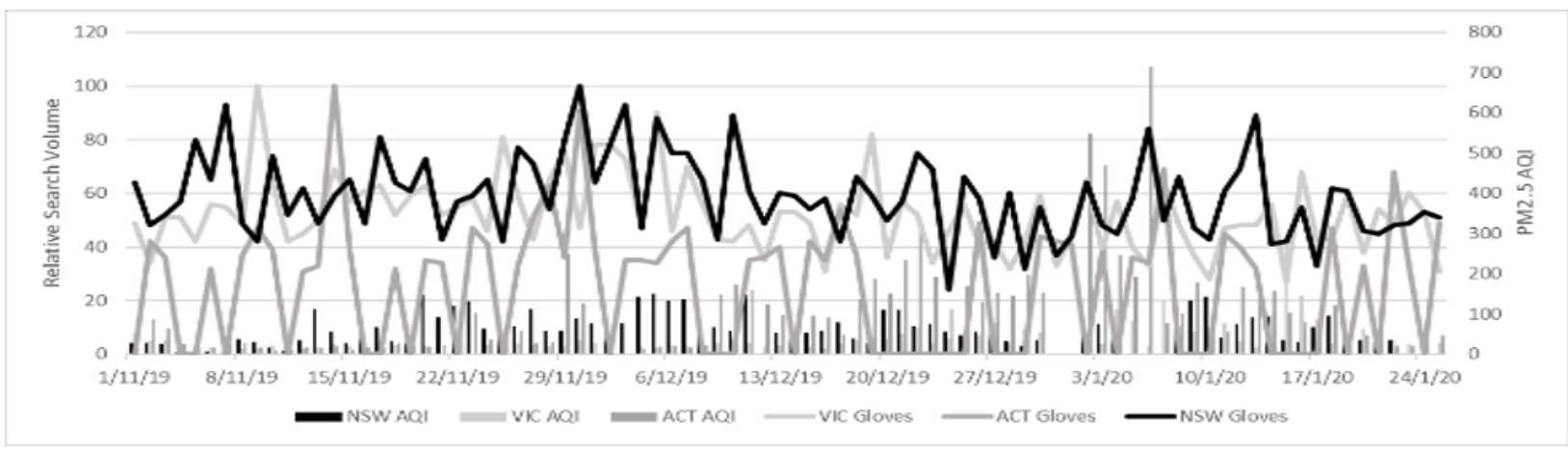

Fig. 7. Gloves RSV by state and event timeline.

Infoveillance trends observed were attributed to changes in observed air quality, not another seasonal event. Table 1 shows Google Trends ${ }^{\mathrm{TM}}$ data for air quality and air purifier significantly correlated to measured air quality during the event $(n=86$ days, Summer 2019-2020) and not the control period $(n=86$ days, Summer 2018-2019). A significant Spearman's correlation only emerged for P2 masks in one location (the ACT) and the control term 'gloves' was not correlated with measured air quality.
Table 1. AQI and RSV CORRELATIONS BY STATE AND TIME.

\begin{tabular}{|llll|}
\hline RSV/Year & $\begin{array}{l}\text { ACT } \\
\boldsymbol{r}\end{array}$ & $\begin{array}{l}\text { NSW } \\
\boldsymbol{r}\end{array}$ & $\begin{array}{l}\text { VIC } \\
\boldsymbol{r}\end{array}$ \\
\hline Gloves & & & \\
\hline 2018-2019 & 0.06 & -0.2 & 0.003 \\
\hline $\mathbf{2 0 1 9 - 2 0 2 0}$ & -0.2 & 0.1 & -0.2 \\
\hline Air purifier & & & \\
\hline $\mathbf{2 0 1 8 - 2 0 1 9}$ & -0.07 & 0.2 & 0.02 \\
\hline $\mathbf{2 0 1 9 - 2 0 2 0}$ & $0.7^{*}$ & $0.5^{*}$ & $0.5^{*}$ \\
\hline Air quality & & & \\
\hline 2018-2019 & -0.07 & 0.02 & -0.02 \\
\hline 2019-2020 & $0.8^{*}$ & $0.5^{*}$ & $0.5^{*}$ \\
\hline P2 mask & & & \\
\hline 2018-2019 & -0.2 & 0.02 & -0.09 \\
\hline 2019 - 2020 & $0.6^{*}$ & 0.4 & 0.3 \\
\hline
\end{tabular}




\section{Discussion}

This infoveillance analysis showed Google Trends ${ }^{\mathrm{TM}}$ data has capacity to reveal, and anticipate, meaningful public science literacy/action-needs patterns in response to the localised experience of emergency events. All three states followed similar public information searching trajectories as each experienced relative to changes in local air quality. This occurred due to the progressive movement of an emergency event, the 2019-2020 Australian summer bushfire. The commonality of internet search activity highlights that once an emergency event starts, authorities and businesses in neighbouring areas may benefit from conducting infoveillance analysis as a preparatory activity. Compared with primary data collection methods, such as surveys or interviews, infoveillance offers an efficient, and arguably accurate, data source to assess public science/health literacy and resource needs, including consumer demand for emergency response products in subsequent locations. Increased local PM2.5 levels not only aligned with increased local internet searching for air quality information, Google Trends ${ }^{\mathrm{TM}}$ data revealed those not yet affected by the event in nearby locations, such as VIC, commenced searching about air quality and air purifiers as neighbouring areas (i.e., NSW) were impacted by smoke. For all states, the increased level of interest in air purifiers was maintained during their period of elevated PM2.5. In this event, consumer interest outpaced industry's capacity to supply air purifiers, with suppliers in each state progressively reporting shortages [26]. Had authorities and/or consumer product suppliers in ACT and VIC conducted infoveillance analysis by using Google Trends ${ }^{\mathrm{TM}}$ data to identify what NSW residents were searching for - to augment their science/health literacy and locate event-mitigating action needs in response to increased PM2.5 levels and external indicators (i.e. increased smoke and media reporting of fires spreading across geographic locations) - then it may have been possible to better predict the likely increased demand for Government-recommended items. Preemptive infoveillance analysis may be particularly useful to ensure there is a reserve of vital emergency resources for the most vulnerable populations (i.e. air purifiers and P2 masks for those with pre-existing respiratory disease, those in remote locations, etc.) by securing supply ahead of consumer demand. Likewise, infoveillance may assist in managing other pollution/disaster events with predicted geographical changes in distribution (i.e. floods, water-based pollution from a point source, pest plagues, etc.).

In addition to lending evidence that Google Trends ${ }^{\mathrm{TM}}$ data has a valuable place in active disaster information management of resource and information provision, findings indicate infoveillance can be useful for measuring and/or addressing community science/health literacy deficits. Air pollution awareness and individual capacity to act, relative to official information provided, are issues traversing health literacy and environmental literacy fields [3]. American research has noted inconsistency in how air pollution information is provided, as well as the general lack of appropriate information available about its risk mitigation and long term impacts [3]. Infoveillance may, in part, offer an accessible and practical approach to identifying public health information and action needs surpassing interest groups and/or intensive research-access restrictions, producing 'snapshots' of time and place specific conditions that may both serve local needs and offer valuable insights from comparative analysis.

In the most recent analysis of air quality monitoring and reporting in an Australian context, the chief auditor of Victoria was highly critical of the paucity of information available to Victorian residents specifically regarding their air quality, noting this predicament was due to a combination of issues from insufficient monitoring across the state to the inadequate quality control of data and selective reporting [4]. While such gaps in information provision require addressing as part of a broader campaign to enhance residents' health literacy in association with scientific air quality monitoring and action-recommendations, enhancing public science and health literacy must be accompanied by improved air quality monitoring in rural and remote locations in Australia; encouraging and empowering the public to review and assess personal health/environmental risks requires reliable and able data which, presently, neither equally nor accurately exists for all Australian residents/citizens since monitoring availability remains contingent upon location's population size [4].

\section{Conclusions}

Nevertheless, by using Google Trends ${ }^{\mathrm{TM}}$ in a manner that is becoming increasingly common in epidemiological studies of human disease utilised in the emerging field of infodemiology through infoveillance [2], this investigation surpasses traditional epidemiology, which focuses on infectious agents, by exploring how individuals use and action government-recommended information to guide personal response to a pollutioninitiated risk during an emergency event. Infoveillance, made possible by the increased digitalisation of society, offers scope for ICT to inform official and community response to environmental pollution events with sizable health costs. The event analysed in this study impacted thousands of individuals over several months across three states, leaving individuals and businesses unprepared and ill protected. Analytically, however, it offers an exemplar of how infoveillance - as a concept and method - may be applicable across academic disciplines and applied areas of specialisation, such as the growing field of pollution control management [27]. Any 'event' or issue involving public/community interaction with ICT related to risks, including risks to government/corporate reputation, may indeed benefit from monitoring avenues where misinformation and/or lack of information can yield deleterious consequences. 


\section{References}

1. K. Balakrishnan, S. Dey, T. Gupta, R.S. Dhaliwal, M. Brauer, A.J. Cohen, J.D. Stanaway, G. Beig, T.K. Joshi, A. N. Aggarwal. Lancet Planet. Health, 3, e26, 2019.

2. G. Eysenbach, J Med Internet Res, 11, p. e11, 2009

3. A.S. Ramírez, S. Ramondt, K. Van Bogart, R. PerezZuniga. J. Health Commun. 24, 75, 2019.

4. Victorian Auditor General, Improving Victoria's Air Quality. 2018.

5. Australian Government Department of the Environment and Energy National Clean Air Agreement. 2019.

6. Victorian Department of Health and Human Services. Coronavirus (COVID-19) daily update. 2020.

7. A. Greenbank, @abcnews. January 14, 2020.

8. World Health Organisation, Ambient air pollution: A global assessment of exposure and burden of disease. 2016.

9. CSIRO, The 2019-20 bushfires: a CSIRO explainer. 2020.

10. A. Remeikis, The Guardian. January 3, 2020.

11. A.N. Borchers, A.J. Palmer, D.M.J.S. Bowman, G.G. Morgan, B.B. Jaludin, F.H. Johnston, Med. J. Aust. (in press). 2020.

12. Statcounter. Search engine market share Australia May 2019-May 2020.

13. A. Crampton, A.T. Ragusa, Rural Soc. 18, 202, 2008.

14. A. Mavragani, G. Ochoa, K.P. Tsagarakis. J. Medical Internet Res. 20, 2018.

15. M. Schootman, A. Toor, P. Cavazos-Rehg, D.B. Jeffe, A. McQueen, J. Eberth, N.O. Davidson. BMJ Open. 5, e006678, 2015.

16. M. Effenberger, A. Kronbichler, J.I. Shin, G. Mayer, H. Tilg, P. Perco. Int. J. Infect. Dis. 95, 192, 2020.

17. J. Ginsberg, M.H. Mohebbi, R.S. Patel, L. Brammer, M.S. Smolinski, L. Brilliant. Nature. 457, 1012, 2009.

18. A. Husnayain, A. Fuad, L. Lazuardi. Glob. Health Action. 12, 1552652, 2019.

19. X. Xu, M. Reed, Perceived pollution and inbound tourism in China. Tour. Manag. Perspect. 21, 109, 2017.

20. D. Nutbeam, Health Promot. Int. 15, 259, 2000.

21. D. Nutbeam, B. McGill, P. Premkumar. Health Promot. Int. 33, 901, 2018.

22. ACT Government. Heavy smoke and hot conditions in ACT. 2020.

23. NSW Government. P2 Masks. 2020

24. NSW Health. Steps to decrease risk from bushfire smoke. 2019.

25. Victoria State Government. Bushfire - guidelines on use of facemasks. 2020.
26. G. Carey, Health Promot. J. Austr. 31, 5, 2020.

27. P.B. Meyer, T.S. Lyons, T.L. Clapp, Projecting environmental trends from economic forecasts. Routledge, UK, 2017. 